\title{
Effective Optimization of Video Transmission in WLAN
}

\author{
${ }^{1}$ Nagesh V, ${ }^{2}$ K.A. Dattreya M.E, M.B.A, (Ph.D) \\ ${ }^{1}$ M.E. Communication systems (ECE) $2^{\text {nd }}$ yr.Adhiyamaan College ofEngineering.,Hosur, India. \\ ${ }^{2}$ Proff, ECE Department, Adhiyamaan College Of Engineering., Hosur, India.
}

\begin{abstract}
The prevalence of high-definition (HD) cameras, televisions, Blu-Ray players, and DVD recorders means that almost all video content is now captured and recorded digitally and much of it in HD. MPEG-2, H.264/AVC, and VC-1 are the most popular codecs in use today, and these rely on decorrelating trans- forms, motion estimation, intra prediction, and variable-length entropy coding $(V L C)$ to achieve good picture quality at high compression ratios. Alongside the need for efficient video compression, there is a critical requirement for error resilience, in particular in association with wireless networks which are characterized by highly dynamic variations in error rate and bandwidth. Compression techniques based on prediction and variablelength coding render an encoded bit stream highly sensitive to channel errors. In the paper, techniques such as pyramid vector quantization ( $P V Q$ ) have been implemented for increasing the ability to prevent error propagation through the use of fixed-length codeword in the Wireless Environment. In the paper, frame performance of the video has been observed in the pyramid vector section which offers greater compression performance in various techniques.
\end{abstract}

Index Terms: Pyramid vector quantization, least significant bit optimization, video coding, wireless video.

\section{Introduction}

Compression forms the backbone for several applications such as storage of images in a database, TV and facsimile transmission, video conferencing. Compression of images involves taking advantage of the redundancy in data present within an image and video. Compression is an essential component in a digital video system and is required to ensure manageable transmitted bitrates and storage. Compression is an essential component in a digital video system and is required to ensure manageable transmitted bitrates and storage. MPEG-2, H.264/AVC, and VC-1 are the most popular codecs in use today, and these rely on decorrelating transforms,

motion estimation, intra prediction, and variable-length entropy coding (VLC) to achieve good picture quality at high compression ratios [1]-[3].

MPEG-2, H.264/AVC, and VC-1 are the most popular codecs in use today, and these rely on decorrelating transforms, motion estimation, intra prediction, and variable-length entropy coding (VLC) to achieve good picture quality at high compression ratios.

The coding redundancy can be reduced using the probability of occurrence of the events (such as graylevel values). To reduce the interpixel redundancy the 2-D pixel array normally used for human viewing and interpretation must be transformed (or mapped) into a more efficient (but not necessarily visible) format. The transformation is called reversible if the original image elements can be reconstructed from the transformed data.

The synthesis filter has been used for the reconstruction of the input image. Video compression can be viewed as the compression of the sequence of the images along side the need for efficient video compression, there is a critical requirement for error resilience, in particular in association with wireless networks which are characterized by highly dynamic variations in error rate and bandwidth.

Video segmentation is useful in many applications for identifying regions of interest in a scene or annotating the data. The MPEG-4 standard needs segmentation for object based video coding. The work on video segmentation include motion based segmentation, spatial segmentation and motion tracking, moving objects extraction, and region growing using spatio-temporal similarity.

In wavelet transform there is no need to block the input image and its basis functions have variable length to avoid blocking artifacts. It is more robust under transmission and decoding errors. It performs better matched to the HVS characteristics. Good frequency resolution at lower frequencies, good time resolution at higher frequencies.

\section{Wavelet Packets}

Ideas of wavelet packet is same as wavelet, the difference is that wavelet packet offers a more complex and flexible analysis because in wavelet packet analysis the lotdetails as well as the approximation are splited. Wavelet packet decomposition gives a lot of base from which we can look for the best representation. 
Wavelet packets represent a generalization of the method of the multi resolution decomposition and comprise the entire family of sub band coded ( tree ) decompositions. It can be characterized as a recursive application of the high pass and low pass filters that form a Quadrature Mirror Filter (QMF) pair. The calculation of the DWT begins by signal filtering by the high pass and low pass filters and then down sampling the output.

The wavelet packet (WP) decomposition the recursive procedure is applied to all the coarse scale approximation an detail signals, which leads to a complete WP tree. The calculation of wavelet packets is often schematically characterized by the formation of a binary tree with each branch representing the high pass and low pass filtered output of a root node. Wavelet packets are efficiently implementable from arbitrary sub band decomposition trees and it may sometimes lead to adaptive wavelet transforms.

The input image $\mathrm{f}(\mathrm{m}, \mathrm{n})$ of resolution $256 \times 256$ is subdivided into low and high frequency bands by performing linear convolution on the gray scale input image with the low pass and high pass FIR filter coefficients.

The down sampled outputs are again subdivided into high and low frequency bands and the above step is repeated. This is continued up to three levels of decomposition i.e. till we are getting eight wavelet packets. Only two levels are shown in Figure1.1.The synthesis filter has been used for the reconstruction of the input image. The up sampled output will be convoluted with the adjacent low pass and high pass synthesizing filters and the outputs will be added up to give the reconstructed output. The careful design of QMFs is very important for achieving good performance in sub band coding.

Wavelet packets offer more flexibility compared to ordinary wavelet based coding. In wavelet packets all the possible tree structure are searched for a particular filter set instead of using a fixed wavelet packet tree and the wavelet packet coefficients of each tree structure have been determined. Then all the wavelet packets from the different tree structures have been carefully investigated for selection of the optimal wavelet packets in order to best match the space frequency characteristics of the input image.

The optimal wavelet packets are selected based on two performance factors.

1. Peak Signal to Noise Ratio (PSNR) of the reconstructed image

2. Compression Ratio.

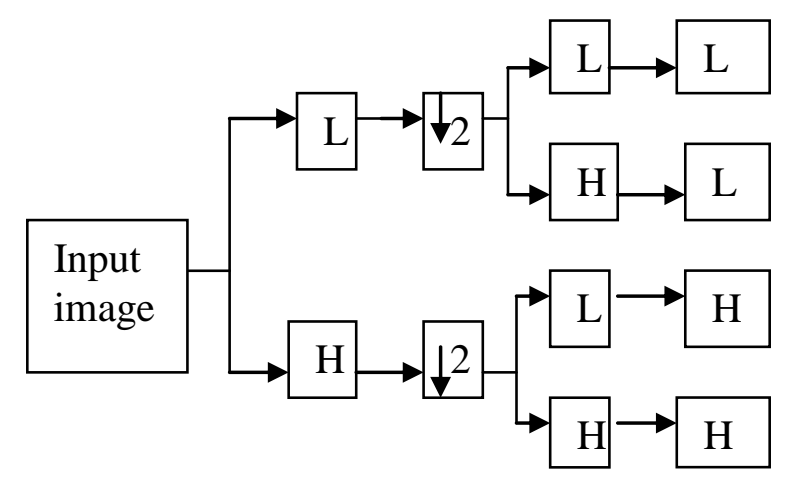

Figure 1.Image Decomposition

\section{Discrete Wavelet Transform}

DWT is a transformation technique is used to represent an image in a new time and frequency scale by decomposing the input image into low frequency, middle and high frequency bands. The value of low frequency band is the averaging value of the filter whereas the high frequency coefficients are wavelet coefficients or detail values.

The DWT can be used to decompose image as a multistage transform. In the first stage, an image is decomposed into four subbands LL1, HL1, LH1, and HH1, where HL1, LH1, and HH1 represent the finest scale wavelet coefficients, while LL1 stands for the coarse level coefficients, i.e., the approximation image. Fig.1.2 shows the one level wavelet decomposition of an image.

\section{PVQ}

Pyramid vector quantization (PVQ) was introduced by Fischer as a fast and efficient method of quantizing Laplacian-like data, such as generated by transforms or sub band filters in an image compression system. PVQ has very simple systematic encoding and decoding algorithms and does not require significant codebook storage. It combines the robustness of fixed-rate codes with the performance of entropy-coded scalar quantization. Considerable research in PVQ algorithms has culminated in high performance, error resilient PVQ image compression systems for both transforms and sub band decompositions. PVQ has also been implemented 
in hardware, taking advantage of a computationally constructed codebook instead of a stored codebook to build a robust, low power, video rate PVQ decoder.

There are new ways of assigning indices to the points in the PVQ codebook that improves channel robustness by up to $3 \mathrm{~dB}$ over previous enumerations, and up to $6 \mathrm{~dB}$ over a randomly enumerated codebook. These new indexing techniques require roughly the same encoding and decoding hardware complexity as previous enumerations and can be applied to any of the fixed rate PVQ coding systems described previously in the literature. We discuss the theoretical and simulated advantage of new techniques through channel noise models of the PVQ indices and codebooks. Finally, we show the practical advantages of PVQ by demonstrating an error-resilient PVQ system that exceeds the performance of Joint Photographic Experts Group (JPEG) implementations, both with and without channel error.

The PVQ-encoded bit stream comprises a sequence of codebook vector indices. These can be generated using techniques such as magnitude enumeration, linear enumeration, conditional product code enumeration, or conditional product- product code enumeration. The application areas include military and surveillance systems where quality and latency are also of paramount importance.

The process of pyramidal vector quantization involves the following steps and the algorithm for pyramidal vector quantization can be listed as

1. All the vectors (having dimension of $\mathrm{L}$ ) taken from the wavelet-transformed images (sub-band images) are projected on the surface of the pyramid $S\left(L, \frac{L}{\lambda}\right)$ such that the projection yields the least mean squared error. All the Vectors lying on the surface of the pyramid $S\left(L, \frac{L}{\lambda}\right)$ have a norm of $\frac{L}{\lambda}$.

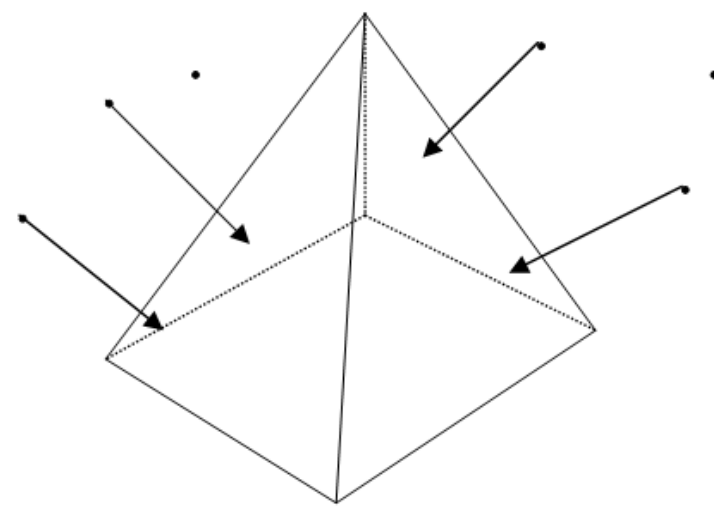

Figure 3.1. Projection of vectors on the surface of the pyramid $S\left(L, \frac{L}{\lambda}\right)$

2. The black dots in space represent the vectors taken from the sub-band images with a dimension of L. These vectors are projected on the surface of the pyramid $S\left(L, \frac{L}{\lambda}\right)$ with least MSE. The main outcome of this projection is that, the dynamic range of the actual vectors is decreased, but produces least distortion.

3. The vectors lying on the surface of the pyramid $S\left(L, \frac{L}{\lambda}\right)$ are then scaled to an inner pyramid $S(L, K)$ with a scaling factor of $\Upsilon$ where the inner pyramid $S(L, K)$ is chosen based on the rate criterion ' $\mathrm{R}$ ' bits/dimension.

Pyramid vector quantization has been previously used for DCT and sub band image compression because both the sub band and transformed image data are similar to the Laplacian in distribution. PVQ also has been considered in the context of matched joint source-channel coding for images, where the channel statistics are stationary and known. The following three sections demonstrate the advantages of the new error-resilient PVQ coding schemes introduced here for sub band image compression under varying channel conditions.

The pyramid vector quantizer, by itself, assigns points to the nearest codeword on a single pyramid shell. For image compression applications where the vector lengths are small, the pyramid vector quantizer is typically matched with a radial quantizer to form several concentric pyramid shells. This is called polar or product quantization, where the radius is quantized independently from the position on the shell. Pyramid vector quantization utilizes four steps to code an input vector into digital bits. 
- The first step is to find the nearest pyramid shell to the input vector.

- The second step is to scale the point onto the cubic lattice.

- The third step is to round the point to the nearest point in the cubic lattice that is on the pyramid shell.

- The fourth step is to enumerate that cubic lattice point, a process which assigns a unique index to each point on the pyramid shell.

In exhaustively examining the radial versus shell quantizer bit allocations, we have found that the radial position index should be allocated about as many bits as the typical sample element in the vector.

This means an overall quantizer resolution of 7 bits per sample element implies that the radial quantizer resolution should be 7 bits. For very large vectors, the radial position index takes up a diminishing fraction of the overall bits; this can be attributed to the asymptotic equipartition principle - for very large vectors, most of the points cluster uniformly on very few shells.

PVQ is an effective codec for indoor wireless transmission of HD video. Intra mode coding was employed since inter frame encoding can lead to temporal error propagation. Although there is a loss in compression performance due to using an intra-only mode, this is more than compensated for by the increased error resilience. Video-optimized multiple input-multiple output (MIMO) WLANs based on IEEE 802.11 are now able to provide the source throughput required by HD intra only codecs.

\section{Simulation And Result Analysis}

The blocks are connected and simulation has to be done. The video input is converted into frames. Each frame is given the sampling rate and decomposed into two sub bands ( high frequency and low frequency). The output is down sampled and it is quantized.

As the simulation starts, the rate of each frame is varied according to the sampling rate and frequency. The varied frames are encoded and it is displayed. Thus the performance of each frame in the video is determined and it is observed accurately.

To understand the superior error resilience of PVQ over H.264/AVC, it is necessary to understand certain aspects of the transmission and decoding processes. In VLC-based schemes such as H.264/AVC, a given frame is divided into slices, where each slice is independently decodable. During transmission, each slice is passed to the physical layer where it is divided into packets, where each packet is independently sent and received. The number of packets PER slice depends on both the slice size and packet size.At the wireless receiver, packets are checked to determine whether or not they contain errors. If a packet is received error-free, it is passed to the application layer (video decoder). However, if it contains errors, the operation performed will beeither:

1) discard the packet and inform the application layer that it has been lost, or;

2) pass the packet to the application layer along with the information that it contains errors.

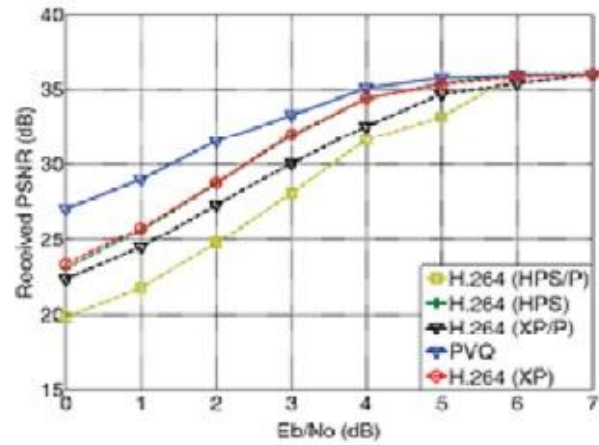

IV. Result

The variation in the each frame is determined and the quantizer encodes the frames individually to the required rate. The PVQ encoder determines the rate of change of video frames. 


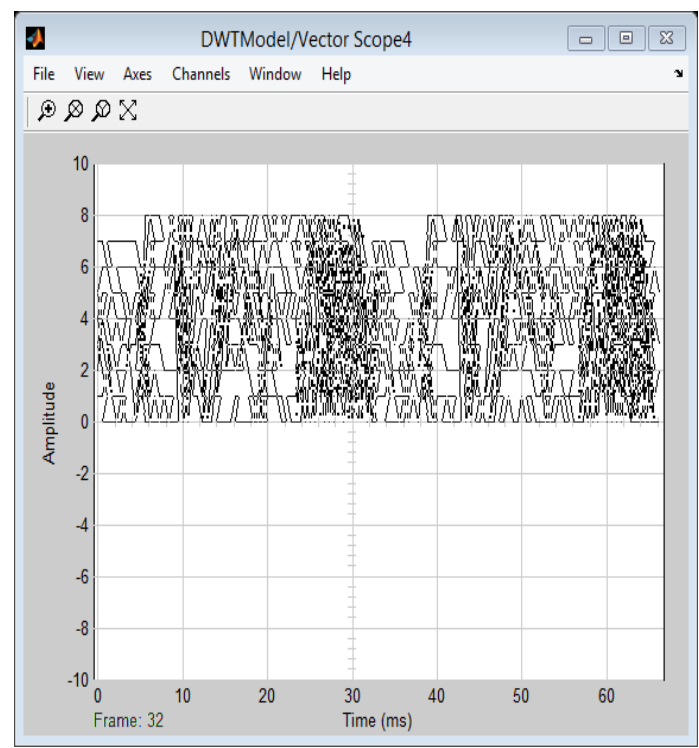

Figure Variation in vector scope 4

\section{Conclusion}

The initial part of the PVQ has been designed with the MATLAB/Simulink successfully. The result shows the better performance and improvement than the existing technique.

The image compression technique based on wavelet packet transform and pyramidal vector quantization is found to perform well with compression ratio and good PSNR values in the range of 20-40 db. The results demonstrate significant gain in percentage zero in wavelet packet with good signal to noise ratio for standard as well as high frequency images. The performance of the proposed scheme was seen to be competitive with that of the state of the art coders in terms of PSNR and Compression ratio.

In terms of error resilience, PVQ (without explicit error resilience tools and without error concealment) has been shown to outperform H.264/AVC (with FMO, slice modes, and error concealment) under a variety of channel, modulation, and coding conditions. For uncorrelated errors, at constant compression bitrates, PVQ offers up to $13.3 \mathrm{db}$ PSNR performance gain over the best performing H.264/AVC counterpart. Even in the case of highly correlated errors, PVQ codec exhibits up to $11.3 \mathrm{~dB}$ PSNR improvement over the best performing H.264/AVC counterpart. PVQ is able to outperform other codecs because of its use of fixed-length coding. This enables the PVQ decoder to extract the maximum utility from corrupted packets.

\section{References}

[1] Chau-Wai Wong and Wan-Chi Siu (2010) Comments on "2-D Order-16 Integer Transforms for HD Video Coding" IEEE Transactions On Circuits And Systems For Video Technology, Vol. X, No. X, pp.1-5.

[2] J. Dong, K. N. Ngan, C.-K. Fong, and W.-K. Cham,(2009) "2-D order-16 integer transforms for HD video coding," IEEE Trans. Circuits Syst. Video Technol., vol. 19, no. 10, pp. 1462-1474.

[3] J. Dong, K. N. Ngan, C.-K. Fong, and W.-K. Cham,(2009) “2-D order-16 Video Technol., vol.19, no. 10, pp. 1462-1474.

[4] T. Fischer, (1986) “A pyramid vector quantizer," IEEE Trans. Inf. Theory,vol. 32, no. 4, pp.568-583.

[5] T. Fischer, (1989) "Geometric source coding and vector quantization," IEEE Trans. Inf. Theory, vol. 35, no. 1, pp. 137-145.

[6] H. Hadizadeh and I. Bajic, (2011) "Burst-loss-resilient packetization of video," IEEE Trans. Image Process., vol. 20, no. 11, pp. 3195-3206. 